\title{
Non-dipole effects in photoelectron angular distributions for rare gas atoms
}

\author{
A. Derevianko and W. R. Johnson \\ Department of Physics, Notre Dame University \\ Notre Dame, IN 46556 \\ K. T. Cheng \\ University of California, Lawrence Livermore National Laboratory \\ Livermore, CA 94550
}

August 25, 1999

\begin{abstract}
We present a theoretical study of $O(k a)$ and $O\left(k^{2} a^{2}\right)$ corrections to the dipole approximation in photoionization of rare gas atoms, where $k$ is the magnitude of the photon wave vector and $a$ is the radius of the ionized subshell. In the dipole approximation, the photoelectron angular distribution is characterized by the single parameter $\beta$. The $O(k a)$ corrections are characterized by two parameters $\gamma$ and $\delta$, and the $O\left(k^{2} a^{2}\right)$ corrections are characterized by three parameters $\lambda, \mu$ and $\nu$, constrained by the relation $\lambda+\mu+\nu=0$ and a correction $\Delta \beta$ to the dipole parameter $\beta$. Formulas are given for the non-dipole parameters in terms of reduced matrix elements of electric and magnetic multipole operators. Tables and graphs of the seven angular distribution parameters, calculated in the relativistic independent-particle approximation (IPA), are given for electron energies ranging from 20-5000 eV for all 41 subshells of the rare gas atoms $\mathrm{He}, \mathrm{Ne}, \mathrm{Ar}, \mathrm{Kr}$, and Xe. Tables and graphs of the $O(k a)$ parameters are also given in the energy range $2-60 \mathrm{eV}$ for the $n=3$ and 4 shell of $\mathrm{Kr}$ and for the $n=4$ and 5 shells of Xe, where interesting non-dipole effects are found. Comparisons of the IPA calculations with correlated relativistic random-phase approximation calculations are made for selected subshells of Ar and Kr illustrating the influence of correlation on the non-dipole parameters.
\end{abstract}

PACS: $32.80 . \mathrm{Fb}$

\section{Contents}

1 Introduction

2 Theory $\quad 3$

$\begin{array}{lll}3 & \text { Relativistic IPA Calculations } & 7\end{array}$

4 Comparison with the Random-Phase Approximation $\quad 8$

4.1 High energies . . . . . . . . . . . . . . . . . . . . . . . . . . . . 9

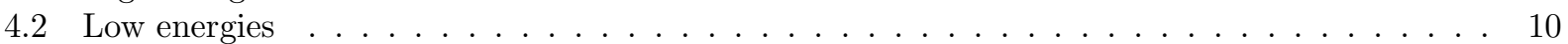




\section{Introduction}

The differential cross section for photoionization of an electron from a subshell of a closed-shell atom by a linearly-polarized photon can be expanded to $O\left(k^{2} a^{2}\right)$, where $k$ is the magnitude of the photon wave vector and $a$ is the radius of the ionized subshell, as

$$
\begin{aligned}
\frac{d \sigma}{d \Omega}(\theta, \phi)=\frac{\sigma}{4 \pi}\{1+(\beta & +\Delta \beta) P_{2}(\cos \theta)+\left(\delta+\gamma \cos ^{2} \theta\right) \sin \theta \cos \phi \\
& \left.+\lambda P_{2}(\cos \theta) \cos 2 \phi+\mu \cos 2 \phi+\nu(1+\cos 2 \phi) P_{4}(\cos \theta)\right\}
\end{aligned}
$$

The parameters $\lambda, \mu$ and $\nu$ are constrained by $\lambda+\mu+\nu=0$. In Eq. (1), the angles $\theta$ and $\phi$ are polar angles of the photoelectron momentum vector $\boldsymbol{p}$ in a coordinate with $z$ axis directed along the polarization vector $\varepsilon$ and $x$ axis directed along the photon propagation direction $\boldsymbol{k}$. To lowest order, $O(1)$, in an expansion in powers of $k a$, the angular distribution takes the dipole form, $1+\beta P_{2}(\cos \theta)$, where the dipole angulardistribution parameter $\beta$ results from interference between electric-dipole amplitudes. The leading $O(k a)$ non-dipole parameters $\delta$ and $\gamma$ arise from interference of electric-dipole amplitudes with electric-quadrupole and magnetic-dipole amplitudes. The magnetic-dipole amplitudes vanish nonrelativistically and are negligible in relativistic calculations for energies below $5000 \mathrm{eV}$. The remaining $O\left(k^{2} a^{2}\right)$ parameters $\Delta \beta, \lambda, \mu$, and $\nu$ result from interference of electric-dipole amplitudes with electric-octopole and magnetic-quadrupole amplitudes, from interference between electric-quadrupole amplitudes, and from retardation corrections to electric-dipole amplitudes.

The $O(k a)$ corrections to the dipole approximation were previously considered in the 1975 review paper of Amusia and Cherepkov [1], who gave formulas for dipole-quadrupole interference contributions to the photoionization differential cross section. In the past decade, there have been a number of theoretical investigations of the $O(k a)$ corrections that include detailed predictions of angular distributions. Notable among these are the calculations of Bechler and Pratt [2], who studied dipole-quadrupole interference effects on the photoelectron angular distribution for $1 s, 2 s$, and $2 p$ shells of elements with nuclear charges $Z$ ranging from 6 to 40 in the Coulomb-field and screened Coulomb-field approximations. Scofield [3] gives general formulas for the interference contributions to the differential cross section and carries out detailed calculations of angular distributions for Ne-like $\mathrm{Ba}$ and He-like Ni using a relativistic independent-particle approximation (IPA) based on the Dirac-Hartree-Slater central potential. The most detailed numerical calculations for neutral atoms are those of Cooper [4], who evaluates the dipole-quadrupole interference corrections nonrelativistically for the inner subshells of all noble-gas atoms from He to Xe in the IPA using a Herman-Skillman potential. Recently, Amusia et al. [5] carried out Hartree-Fock (HF) and random-phase approximation (RPA) calculations of the non-dipole parameter $\gamma$ for the $1 s$ and $3 s$ subshells of Argon at low energies. Large non-dipole effects were found and correlation corrections to $\gamma$ were found to be significant for these cases.

Measurements of the non-dipole angular-distribution parameters for inner shells of Ar and $\mathrm{Kr}$, reported in Refs. [6] and [7], are in excellent agreement with IPA calculations; however, measurements of $\gamma+3 \delta$ for the $2 p$ subshells of Ne reported in [8] are systematically higher than the IPA predictions for energies above the Ne $1 s$ threshold. In Ref. [9], random-phase approximation calculations were carried out to investigate the possible influence of correlation on the non-dipole parameters. These calculations confirmed a large intershell coupling effect on the dipole parameter $\beta$ observed experimentally, but failed to explain the substantial differences between IPA calculations and experiment for the non-dipole parameter $\gamma+3 \delta$. Indeed, the RPA and IPA 
values for the non-dipole parameter were found to be in close agreement with one another. The differences between theory and experiment for the $n=2$ shell of neon were subsequently shown to arise from corrections of $O\left(k^{2} a^{2}\right)$ and completely resolved in Ref. [10].

In Table I, we present the results of systematic calculations of the $O(1)$ angular distribution parameter $\beta$, the $O(k a)$ parameters $\gamma$ and $\delta$, and the $O\left(k^{2} a^{2}\right)$ parameters $\Delta \beta, \lambda, \mu$, and $\nu$, for all 41 subshells of the rare gas atoms in the electron energy range $20-5000 \mathrm{eV}$. These calculations are carried out in the relativistic IPA using a modified Hartree potential. We use the relativistic theory in order to account for the fine-structure separation between atomic subshells. Numerical results are given in both tabular and graphical forms. Our aim is to provide a database that can be used to design future experiments. Comparisons of experimental measurements with the IPA data will also be helpful in improving theoretical models of photoionization. The present results for the $O(k a)$ parameters $\gamma$ and $\delta$ are in fair agreement with previous calculations of Cooper [4], but provide more detail, especially in the low-energy region. Results of the $O\left(k^{2} a^{2}\right)$ parameters $\Delta \beta, \lambda, \mu$ and $\nu$ for the $n=2$ shell of neon were given previously in [10]; values of the $O\left(k^{2} a^{2}\right)$ parameters for other cases have not been published previously.

In Table II, we present values of $\beta, \delta$ and $\gamma$ for the $n=3$ and 4 shells of $\mathrm{Kr}$ and for the $n=4$ and 5 shells of $\mathrm{Xe}$ in the low electron energy range $2-60 \mathrm{eV}$. For some of these subshells, surprisingly large non-dipole effects are found.

To assess the importance of correlation corrections to the non-dipole parameters, we carry out relativistic random-phase approximation (RRPA) [11] calculations for selected subshells of Ar, Kr, and Xe. For photoelectron energies above $100 \mathrm{eV}$, we find close agreement between theoretical IPA parameters from Table I and those obtained from the RRPA. Moreover, the theoretical parameters are in excellent agreement with available experimental data. For photoelectron energies below $50 \mathrm{eV}$, where correlation effects are expected to be more important, we find substantial modifications of the IPA values of the angular distribution parameters given in Table II near "Cooper" minima (where the dominant dipole amplitude vanishes) of outer $n s$ subshells, otherwise the IPA and RRPA calculations are found to be in good agreement.

\section{Theory}

We designate multipole operators in a spherical basis by $q_{J M}^{(\lambda)}$, where $\lambda=1$ for electric multipoles and $\lambda=0$ for magnetic multipoles, and we introduce the single-particle reduced multipole matrix elements between a continuum state $\epsilon \kappa$ and a bound state $n_{b} \kappa_{b}$

$$
\mathcal{Q}_{J}^{(\lambda)}\left(\kappa, \kappa_{b}\right)=i^{-l+1} e^{i \delta_{\kappa}}\left\langle\epsilon \kappa\left\|q_{J}^{(\lambda)}\right\| n_{b} \kappa_{b}\right\rangle .
$$

Here, $\delta_{\kappa}$ is the phase shift of the continuum state and $l$ is the orbital angular momentum of the continuum state. For convenience, we introduce the following notation for the lowest few multipole matrix elements:

$$
\begin{array}{ll}
\mathcal{D}_{\kappa}=\mathcal{Q}_{1}^{(1)}\left(\kappa, \kappa_{b}\right) & \text { (electric dipole) } \\
\mathcal{M}_{\kappa}=\mathcal{Q}_{1}^{(0)}\left(\kappa, \kappa_{b}\right) & \text { (magnetic dipole) } \\
\mathcal{Q}_{\kappa}=\mathcal{Q}_{2}^{(1)}\left(\kappa, \kappa_{b}\right) & \text { (electric quadrupole) } \\
\mathcal{N}_{\kappa}=\mathcal{Q}_{2}^{(0)}\left(\kappa, \kappa_{b}\right) & \text { (magnetic quadrupole) } \\
\mathcal{O}_{\kappa}=\mathcal{Q}_{3}^{(1)}\left(\kappa, \kappa_{b}\right) & \text { (electric octopole). }
\end{array}
$$

With these definitions, we can write the contributions to $O\left(k^{2} a^{2}\right)$ to the cross section in the form

$$
\sigma(\omega)=\frac{4 \pi^{2} \alpha}{3} \omega \bar{\sigma}
$$



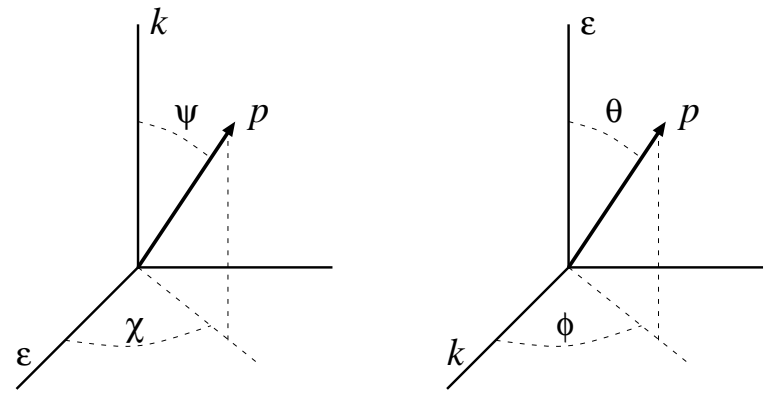

Figure 1: Comparison of coordinate systems.

where

$$
\bar{\sigma}=\sum_{\kappa}\left[\left|\mathcal{D}_{\kappa}\right|^{2}+\left|\mathcal{M}_{\kappa}\right|^{2}+\frac{k^{2}}{20}\left|\mathcal{Q}_{\kappa}\right|^{2}\right] .
$$

The photoelectron angular distribution can be written

$$
\frac{d \sigma}{d \Omega}=\frac{\sigma}{4 \pi}[1+\mathcal{A}(\psi, \chi)],
$$

where $(\psi, \chi)$ are polar angles of $\boldsymbol{p}$ in a coordinate system with $\boldsymbol{k}$ along the $z$ axis and $\boldsymbol{\varepsilon}$ along the $x$ axis. In Fig. 1, we compare this coordinate system with the $(\theta, \phi)$ coordinate system used earlier in Eq. (1). The angular factor $\mathcal{A}(\psi, \chi)$ may be expressed as a sum over multipole interference terms

$$
\mathcal{A}(\psi, \chi)=\sum_{m n L} \mathcal{A}_{P_{m} P_{n}}^{(L)}(\psi, \chi),
$$

where the interference terms are given by

$$
\begin{aligned}
\mathcal{A}_{E_{1} E_{1}}^{(2)}(\psi, \chi) & =-\frac{1}{2} \beta\left[P_{2}(\cos \psi)-\frac{3}{2} \cos 2 \chi \sin ^{2} \psi\right] \\
\beta & =\frac{1}{\bar{\sigma}} \sum_{\kappa \kappa^{\prime}} \sqrt{30}\left\langle\kappa^{\prime}\left\|C_{2}\right\| \kappa\right\rangle(-1)^{j^{\prime}+j_{b}}\left\{\begin{array}{lll}
1 & 1 & 2 \\
j^{\prime} & j & j_{b}
\end{array}\right\} \Re\left[\mathcal{D}_{\kappa} \mathcal{D}_{\kappa^{\prime}}^{*}\right], \\
\mathcal{A}_{E_{1} M_{1}}^{(1)}(\psi, \chi) & =\Delta_{1} P_{1}(\cos \psi), \\
\Delta_{1} & =\frac{1}{\bar{\sigma}} \sum_{\kappa \kappa^{\prime}}-3 \sqrt{6}\left\langle\kappa^{\prime}\left\|C_{1}\right\| \kappa\right\rangle(-1)^{j^{\prime}+j_{b}}\left\{\begin{array}{ccc}
1 & 1 & 1 \\
j^{\prime} & j & j_{b}
\end{array}\right\} \Im\left[\mathcal{D}_{\kappa} \mathcal{M}_{\kappa^{\prime}}^{*}\right], \\
\mathcal{A}_{E_{1} E_{2}}^{(1)}(\psi, \chi) & =\Gamma_{1} P_{1}(\cos \psi), \\
\Gamma_{1} & =\frac{k}{\bar{\sigma}} \sum_{\kappa \kappa^{\prime}} \frac{3}{10} \sqrt{30}\left\langle\kappa^{\prime}\left\|C_{1}\right\| \kappa\right\rangle(-1)^{j^{\prime}+j_{b}}\left\{\begin{array}{lll}
1 & 2 & 1 \\
j^{\prime} & j & j_{b}
\end{array}\right\} \Im\left[\mathcal{D}_{\kappa} \mathcal{Q}_{\kappa^{\prime}}^{*}\right], \\
\mathcal{A}_{E_{1} E_{2}}^{(3)}(\psi, \chi) & =\Gamma_{3}\left[P_{3}(\cos \psi)-\frac{5}{2} \cos 2 \chi \cos \psi \sin ^{2} \psi\right], \\
\Gamma_{3} & =\frac{k}{\bar{\sigma}} \sum_{\kappa \kappa^{\prime}} \frac{1}{5} \sqrt{105}\left\langle\kappa^{\prime}\left\|C_{3}\right\| \kappa\right\rangle(-1)^{j^{\prime}+j_{b}}\left\{\begin{array}{ccc}
1 & 2 & 3 \\
j^{\prime} & j & j_{b}
\end{array}\right\} \Im\left[\mathcal{D}_{\kappa} \mathcal{Q}_{\kappa^{\prime}}^{*}\right],
\end{aligned}
$$




$$
\begin{aligned}
& \mathcal{A}_{E_{1} M_{2}}^{(2)}(\psi, \chi)=\Upsilon_{2}\left[P_{2}(\cos \psi)+\frac{1}{2} \cos 2 \chi \sin ^{2} \psi\right], \\
& \Upsilon_{2}=\frac{k}{\bar{\sigma}} \sum_{\kappa \kappa^{\prime}}-\frac{1}{2} \sqrt{30}\left\langle\kappa^{\prime}\left\|C_{2}\right\| \kappa\right\rangle(-1)^{j^{\prime}+j_{b}}\left\{\begin{array}{ccc}
1 & 2 & 2 \\
j^{\prime} & j & j_{b}
\end{array}\right\} \Re\left[\mathcal{D}_{\kappa} \mathcal{N}_{\kappa^{\prime}}^{*}\right], \\
& \mathcal{A}_{E_{1} E_{3}}^{(2)}(\psi, \chi)=\Lambda_{2}\left[P_{2}(\cos \psi)-\frac{1}{4} \cos 2 \chi \sin ^{2} \psi\right] \text {, } \\
& \Lambda_{2}=\frac{k^{2}}{\bar{\sigma}} \sum_{\kappa \kappa^{\prime}} \frac{4}{105} \sqrt{105}\left\langle\kappa^{\prime}\left\|C_{2}\right\| \kappa\right\rangle(-1)^{j^{\prime}+j_{b}}\left\{\begin{array}{lll}
1 & 3 & 2 \\
j^{\prime} & j & j_{b}
\end{array}\right\} \Re\left[\mathcal{D}_{\kappa} \mathcal{O}_{\kappa^{\prime}}^{*}\right] \\
& \mathcal{A}_{E_{1} E_{3}}^{(4)}(\psi, \chi)=\Lambda_{4}\left[P_{4}(\cos \psi)-\frac{5}{8}\left(7 \cos ^{2} \psi-1\right) \sin ^{2} \psi \cos 2 \chi\right] \text {, } \\
& \Lambda_{4}=\frac{k^{2}}{\bar{\sigma}} \sum_{\kappa \kappa^{\prime}} \frac{6}{35} \sqrt{7}\left\langle\kappa^{\prime}\left\|C_{4}\right\| \kappa\right\rangle(-1)^{j^{\prime}+j_{b}}\left\{\begin{array}{ccc}
1 & 3 & 4 \\
j^{\prime} & j & j_{b}
\end{array}\right\} \Re\left[\mathcal{D}_{\kappa} \mathcal{O}_{\kappa^{\prime}}^{*}\right], \\
& \mathcal{A}_{E_{2} E_{2}}^{(2)}(\psi, \chi)=\Pi_{2}\left[P_{2}(\cos \psi)+\frac{3}{2} \cos 2 \chi \sin ^{2} \psi\right] \text {, } \\
& \Pi_{2}=\frac{k^{2}}{\bar{\sigma}} \sum_{\kappa \kappa^{\prime}}-\frac{1}{56} \sqrt{70}\left\langle\kappa^{\prime}\left\|C_{2}\right\| \kappa\right\rangle(-1)^{j^{\prime}+j_{b}}\left\{\begin{array}{ccc}
2 & 2 & 2 \\
j^{\prime} & j & j_{b}
\end{array}\right\} \Re\left[\mathcal{Q}_{\kappa} \mathcal{Q}_{\kappa^{\prime}}^{*}\right], \\
& \mathcal{A}_{E_{2} E_{2}}^{(4)}(\psi, \chi)=\Pi_{4}\left[P_{4}(\cos \psi)-\frac{5}{8}\left(7 \cos ^{2} \psi-1\right) \sin ^{2} \psi \cos 2 \chi\right], \\
& \Pi_{4}=\frac{k^{2}}{\bar{\sigma}} \sum_{\kappa \kappa^{\prime}}-\frac{3}{70} \sqrt{70}\left\langle\kappa^{\prime}\left\|C_{4}\right\| \kappa\right\rangle(-1)^{j^{\prime}+j_{b}}\left\{\begin{array}{ccc}
2 & 2 & 4 \\
j^{\prime} & j & j_{b}
\end{array}\right\} \Re\left[\mathcal{Q}_{\kappa} \mathcal{Q}_{\kappa^{\prime}}^{*}\right] .
\end{aligned}
$$

In the above equations, $\Re[X]$ and $\Im[X]$ designate the real and imaginary parts of the argument $X$, respectively, and $\left\langle\kappa^{\prime}\left\|C_{k}\right\| \kappa\right\rangle$ is the reduced matrix element of the spherical tensor

$$
C_{k q}(\hat{r})=\sqrt{\frac{4 \pi}{2 k+1}} Y_{k q}(\hat{r}) .
$$

We retain the tiny $O(k a)$ contribution from $E_{1}-M_{1}$ interference in the present calculation, but we ignore the $O\left(k^{2} a^{2}\right)$ contributions from $M_{1}-E_{2}$ and $M_{1}-M_{1}$ interference. We summarize the above formulas as:

$$
\begin{aligned}
\mathcal{A}(\psi, \chi) & =\left[\Delta_{1}+\Gamma_{1}\right] P_{1}(\cos \psi)+\left[-\frac{1}{2} \beta+\Upsilon_{2}+\Lambda_{2}+\Pi_{2}\right] P_{2}(\cos \psi) \\
& +\Gamma_{3} P_{3}(\cos \psi)+\left[\Lambda_{4}+\Pi_{4}\right] P_{4}(\cos \psi) \\
& +\left[\frac{3}{4} \beta+\frac{1}{2} \Upsilon_{2}-\frac{1}{4} \Lambda_{2}+\frac{3}{2} \Pi_{2}-\frac{5}{2} \Gamma_{3} \cos \psi\right] \sin ^{2} \psi \cos 2 \chi \\
& -\left[\frac{5}{8} \Lambda_{4}+\frac{5}{8} \Pi_{4}\right]\left(7 \cos ^{2} \psi-1\right) \sin ^{2} \psi \cos 2 \chi
\end{aligned}
$$

The following equations are used to transform from the coordinates $(\psi, \chi)$ to the coordinates $(\theta, \phi)$ used in Eq.(1):

$$
\sin \psi \cos \chi=\cos \theta
$$




$$
\begin{aligned}
\sin \psi \sin \chi & =-\sin \theta \sin \phi \\
\cos \psi & =\sin \theta \cos \phi .
\end{aligned}
$$

With the aid of these relations, we can re-express the angular distribution function $\mathcal{A}$ in terms of coordinates $(\theta, \phi)$ as:

$$
\begin{aligned}
\mathcal{A}(\theta, \phi)= & {\left[\beta-\frac{3}{4} \Lambda_{2}+\Pi_{2}\right] P_{2}(\cos \theta) } \\
& +\left[\left(\Delta_{1}+\Gamma_{1}+\Gamma_{3}\right)-5 \Gamma_{3} \cos ^{2} \theta\right] \sin \theta \cos \phi \\
& +\left[-\frac{2}{3} \Upsilon_{2}-\frac{5}{12} \Lambda_{2}-\Pi_{2}-\frac{5}{6}\left(\Lambda_{4}+\Pi_{4}\right)\right] P_{2}(\cos \theta) \cos 2 \phi \\
& +\left[\frac{2}{3} \Upsilon_{2}+\frac{5}{12} \Lambda_{2}+\Pi_{2}-\frac{1}{6}\left(\Lambda_{4}+\Pi_{4}\right)\right] \cos 2 \phi \\
& +\left[\Lambda_{4}+\Pi_{4}\right](1+\cos 2 \phi) P_{4}(\cos \theta),
\end{aligned}
$$

or alternatively,

$$
\begin{aligned}
\mathcal{A}(\theta, \phi)= & (\beta+\Delta \beta) P_{2}(\cos \theta)+\left(\delta+\gamma \cos ^{2} \theta\right) \sin \theta \cos \phi \\
& +\lambda P_{2}(\cos \theta) \cos 2 \phi+\mu \cos 2 \phi+\nu(1+\cos 2 \phi) P_{4}(\cos \theta) .
\end{aligned}
$$

The terms of $O(k a)$ in Eq. (18) are

$$
\begin{aligned}
\delta & =\Delta_{1}+\Gamma_{1}+\Gamma_{3}, \\
\gamma & =-5 \Gamma_{3} .
\end{aligned}
$$

The $M_{1}$ amplitude, and therefore $\Delta_{1}$, vanishes nonrelativistically. Furthermore, in the nonrelativistic limit $\Gamma_{3}=-\Gamma_{1}$ for photoionization of $n s$ subshells; consequently, $\delta$ vanishes in these subshells nonrelativistically. In the present relativistic calculations, $\delta$ is nonvanishing but negligibly small for $n s$ subshells for energies below $5000 \mathrm{eV}$.

The terms of $O\left(k^{2} a^{2}\right)$ in Eq. (18) are

$$
\begin{aligned}
\Delta \beta & =-\frac{3}{4} \Lambda_{2}+\Pi_{2}, \\
\lambda & =-\frac{2}{3} \Upsilon_{2}-\frac{5}{12} \Lambda_{2}-\Pi_{2}-\frac{5}{6}\left(\Lambda_{4}+\Pi_{4}\right), \\
\mu & =\frac{2}{3} \Upsilon_{2}+\frac{5}{12} \Lambda_{2}+\Pi_{2}-\frac{1}{6}\left(\Lambda_{4}+\Pi_{4}\right), \\
\nu & =\Lambda_{4}+\Pi_{4} .
\end{aligned}
$$

From the latter three equations, it follows that

$$
\lambda+\mu+\nu=0 .
$$

In our numerical studies, we include retardation in the electric-dipole amplitudes, but ignore it elsewhere. We thereby account for all $O\left(k^{2} a^{2}\right)$ corrections. 
The cross section for unpolarized photoionization is written in the form

$$
\left.\frac{d \sigma}{d \Omega}\right|_{\text {unpol }}=\frac{\sigma}{4 \pi}\left[1+\sum_{l=1}^{\infty} B_{l} P_{l}(\cos \psi)\right],
$$

by Bechler and Pratt in Ref. [2] and by Scofield in Ref. [3]. Comparing Eqs.(16) and (26), we find that the parameters $B_{k}$ are related to our coefficients by

$$
\begin{aligned}
B_{1} & =\Delta_{1}+\Gamma_{1}=\delta+\frac{1}{5} \gamma, \\
B_{2} & =-\frac{1}{2} \beta+\Upsilon_{2}+\Lambda_{2}+\Pi_{2}=-\frac{1}{2}[\beta+\Delta \beta]+\frac{1}{4}[5 \mu-\lambda], \\
B_{3} & =\Gamma_{3}=-\frac{1}{5} \gamma, \\
B_{4} & =\Lambda_{4}+\Pi_{4}=\nu .
\end{aligned}
$$

Amusia and Cherepkov [1] consider $E_{1}-E_{2}$ interference effects and express the unpolarized cross section in the form

$$
\left.\frac{d \sigma}{d \Omega}\right|_{\text {unpol }}=\frac{\sigma}{4 \pi}\left[1-\frac{1}{2} \beta P_{2}(\cos \psi)+k \gamma^{\prime} P_{1}(\cos \psi)+k \eta^{\prime} P_{3}(\cos \psi)\right] .
$$

The parameters $k \gamma^{\prime}$ and $k \eta^{\prime}$ are related to our parameters by

$$
\begin{aligned}
k \gamma^{\prime} & =\Gamma_{1}=\delta+\frac{1}{5} \gamma, \\
k \eta^{\prime} & =\Gamma_{3}=-\frac{1}{5} \gamma .
\end{aligned}
$$

\section{Relativistic IPA Calculations}

The present relativistic IPA calculations of the angular-distribution parameters are carried out using wave functions obtained by solving the Dirac equation in a modified Hartree potential. For a closed-shell $\mathrm{N}$ electron atom, this potential is defined by

$$
V(r)=\sum_{b}\left(2 j_{b}+1\right) \frac{Y_{0}(b, r)}{r}-V_{\mathrm{exc}}(r) .
$$

The sum in the first term, which gives the direct part of the potential, ranges over all occupied subshells. The function $Y_{0}(b, r) / r$ is the Hartree screening potential for an electron in subshell $b$, and the exchange potential $V_{\text {exc }}(r)$ is given as $1 / N$ of the direct potential. As in the Dirac-Hartree-Fock theory, the orbitals calculated in this modified Hartree potential are orthonormal. We solve the radial Dirac-Hartree equation (self-consistently) to obtain the bound-state orbitals $\phi_{b}(r)$, bound-state energies $E_{b}$, and the potential $V(r)$. The continuum orbitals are then obtained by solving the Dirac equation in the same potential.

The non-dipole angular-distribution parameters, expressed as functions of electron energy $E$, are insensitive to the potential $V(r)$. Values of the $O(k a)$ parameters $\gamma$ and $\delta$ from the present calculation are in excellent agreement with values from the relativistic screened Coulomb field calculations of Ref. [2] as well as values from the nonrelativistic Herman-Skillman calculations of Ref. [4]. However, theoretical binding 
Table A. Inner-shell binding energies $E_{b}(\mathrm{eV})$ and references to data sources.

\begin{tabular}{|c|c|c|c|c|c|c|c|c|c|c|c|}
\hline \multicolumn{2}{|c|}{ Shell } & $\mathrm{He}$ & Ref. & $\overline{\mathrm{Ne}}$ & Ref. & $\mathrm{Ar}$ & Ref. & $\mathrm{Kr}$ & Ref. & $\mathrm{Xe}$ & Ref. \\
\hline $\bar{K}$ & $1 s$ & 24.6 & {$[12]$} & 870.2 & {$[13]$} & 3206.1 & {$[13]$} & 14327.1 & {$[13]$} & 34565.2 & {$[13]$} \\
\hline $\mathrm{L}_{I}$ & $2 s$ & & & 53.0 & {$[14]$} & 327.3 & [14] & 1920.4 & [13] & 5452.6 & [13] \\
\hline $\mathrm{L}_{I I}$ & $2 p_{1 / 2}$ & & & 21.7 & {$[12]$} & 251.6 & {$[14]$} & 1730.9 & [13] & 5106.7 & [13] \\
\hline $\mathrm{L}_{I I I}$ & $2 p_{3 / 2}$ & & & 21.6 & {$[12]$} & 249.5 & {$[14]$} & 1679.1 & {$[13]$} & 4786.5 & [13] \\
\hline $\mathrm{M}_{I}$ & $3 s$ & & & & & 30.0 & [14] & 293.1 & [14] & 1150.5 & [14] \\
\hline $\mathrm{M}_{I I}$ & $3 p_{1 / 2}$ & & & & & 15.9 & {$[12]$} & 222.4 & {$[14]$} & 1003.5 & [14] \\
\hline $\mathrm{M}_{I I I}$ & $3 p_{3 / 2}$ & & & & & 15.8 & {$[12]$} & 214.8 & [14] & 941.9 & [14] \\
\hline $\mathrm{M}_{I V}$ & $3 d_{3 / 2}$ & & & & & & & 95.4 & [14] & 690.5 & [14] \\
\hline $\mathrm{M}_{V}$ & $3 d_{5 / 2}$ & & & & & & & 94.2 & [14] & 677.9 & [14] \\
\hline $\mathrm{N}_{I}$ & $4 s$ & & & & & & & 27.5 & {$[15]$} & 217.7 & [15] \\
\hline $\mathrm{N}_{I I}$ & $4 p_{1 / 2}$ & & & & & & & 14.7 & {$[12]$} & 163.9 & [15] \\
\hline $\mathrm{N}_{I I I}$ & $4 p_{3 / 2}$ & & & & & & & 14.0 & [12] & 156.5 & [15] \\
\hline $\mathrm{N}_{I V}$ & $4 d_{3 / 2}$ & & & & & & & & & 69.5 & [15] \\
\hline $\mathrm{N}_{V}$ & $4 d_{5 / 2}$ & & & & & & & & & 67.6 & [15] \\
\hline $\mathrm{O}_{I}$ & $5 s$ & & & & & & & & & 23.4 & [15] \\
\hline $\mathrm{O}_{I I}$ & $5 p_{1 / 2}$ & & & & & & & & & 13.4 & [12] \\
\hline $\mathrm{O}_{I I I}$ & $5 p_{3 / 2}$ & & & & & & & & & 12.1 & [12] \\
\hline
\end{tabular}

energies $E_{b}$ obtained using different potentials differ from one another and from the experimental binding energies. As a consequence, the angular- distribution parameters, expressed as functions of the theoretical photon energy $\omega=E+E_{b}$, depend on the potential. In the Tables I and II, we express the angulardistribution parameters as functions of the photoelectron energy $E$ to reduce the sensitivity to the starting potential, and we use the most accurate available values of $E_{b}$ to determine the corresponding values of photon energy $\omega$ :

1. For the two outermost $n p_{1 / 2}$ and $n p_{3 / 2}$ subshells of the rare gas atoms, we use spectroscopic values of the binding energies from Moore [12].

2. For the $\mathrm{K}$ shells of $\mathrm{Ne}, \mathrm{Ar}, \mathrm{Kr}$ and $\mathrm{Xe}$, and for the $\mathrm{L}$ shells of $\mathrm{Kr}$ and $\mathrm{Xe}$, we use a recent reevaluation of the inner-shell binding energies by Deslattes et al. [13].

3. Otherwise, for $\mathrm{L}$ and $\mathrm{M}$ shells, we use the precise theoretical values of the binding energies from the many-body calculations of Indelicato, Boucard and Lindroth [14].

4. Finally, for the remaining $\mathrm{N}$ subshells of krypton and xenon, and the $\mathrm{O}_{I}$ subshell of xenon, we use experimental values of the binding energies from the tables of Sevier [15].

Values of $E_{b}$ used here and their sources are given in Table A; the values are repeated in the headings of Tables I and II.

\section{Comparison with the Random-Phase Approximation}

Although there is good consistency between IPA calculations starting from different potentials, it is nevertheless important to investigate the influence of electron correlation on the non-dipole parameters, especially at 

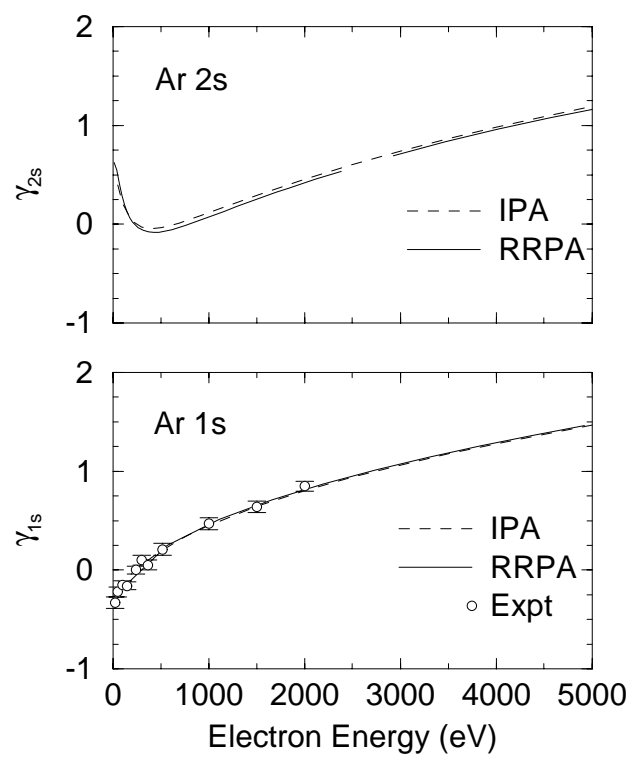

Figure 2: Comparison of IPA and RRPA calculations of $\gamma$ for the $1 s$ and $2 s$ shells of argon with one another and with experiment $[6]$.

lower energies, where correlation corrections to cross sections and $\beta$-parameters are known to be significant. For this purpose, we employ the relativistic random-phase approximation (RRPA) described in Refs. [11]. The RRPA starts from the Dirac-Hartree-Fock approximation and includes lowest-order correlation corrections together with those higher-order corrections obtained by iterating the lowest-order ones. Partial cross sections, dipole angular-distribution parameters, and spin-polarization parameters for the outer shells of Ar, $\mathrm{Kr}$, and Xe calculated in the RRPA were published in Ref. [16].

The RRPA calculations are significantly more complex than the IPA calculations since the photoionization amplitudes from individual atomic subshells are coupled. Thus, in our RRPA calculations of the electric dipole photoionization amplitudes for Ar, all dipole excitations from $1 s, 2 s$ and $3 s$ subshells to continuum $p$ states, and from the $2 p$ and $3 p$ subshells to continuum $s$ and $d$ states are included. This leads to a coupled 16-channel problem. Similarly, the RRPA calculation of electric quadrupole amplitudes leads to an 18-channel problem, in which $1 s, 2 s$ and $3 s$ subshells are excited to continuum $d$ states, and $2 p$ and $3 p$ subshells are excited to continuum $p$ and $f$ states. It should be noted that, as in the IPA, the dipole and quadrupole transition amplitudes calculated using the RRPA are independent of gauge, so length-form and velocity-form amplitudes are identical. We neglect the tiny magnetic-dipole contributions in the present RRPA calculations.

\subsection{High energies}

In Fig. 2, we compare IPA values of the $O(k a)$ parameter $\gamma$ for the $1 s$ and $2 s$ shells of Ar given in Table I with RRPA values and with experimental results from [6]. The effects of correlation are seen to be insignificant for both cases and the theoretical calculations agree well with measurements. In Fig. 3, we make similar 

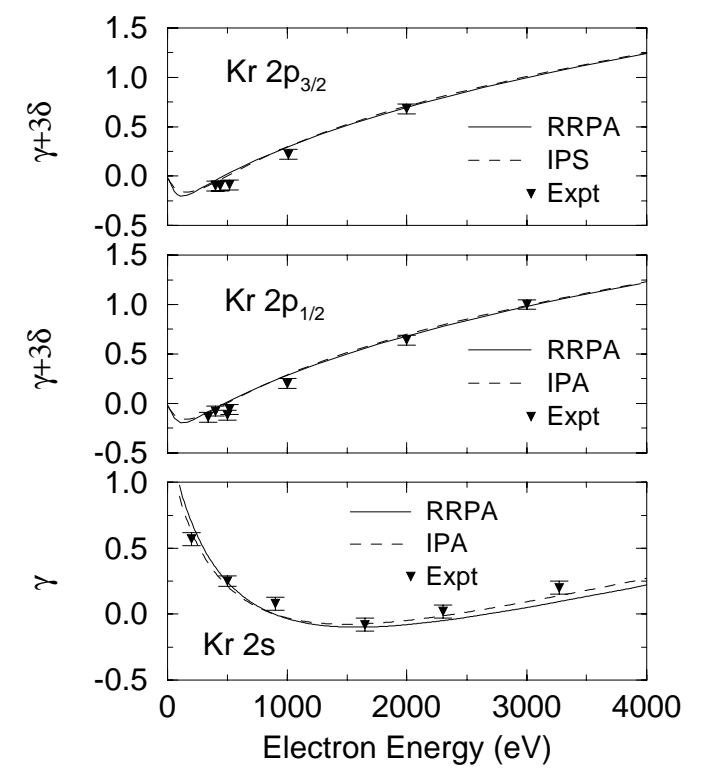

Figure 3: Comparison of IPA and RRPA calculations of $\gamma$ for the $2 s$ subshell and $\gamma+3 \delta$ for the $2 p$ subshells shells of krypton with one another and with experiment [7].

comparisons of $\gamma$ for the $2 s$ subshell of $\mathrm{Kr}$ and $\gamma+3 \delta$ for the $2 p_{1 / 2}$ and $2 p_{3 / 2}$ subshells of Kr. Again, agreement between theory and experiment is excellent. Differences between IPA values given in Table I and RRPA calculations, which measure the size of correlation effects, are seen to be insignificant. Moreover, when considered as functions of electron energy, values of the non-dipole parameters for the $2 p_{1 / 2}$ and $2 p_{3 / 2}$ subshells are in close agreement. For the case of neon, correlation effects on $\gamma+3 \delta$ for the $2 p$ subshells in the $1000 \mathrm{eV}$ energy range were studied in Ref. [9] using both the non-relativistic RPAE [1] and the RRPA and also found to be unimportant.

\subsection{Low energies}

Since large correlation effects for the $3 s$ subshell of Ar were found near threshold in Ref. [5], it is particularly interesting to compare IPA and RRPA calculations for low electron energies. In the lower panel of Fig. 4, we compare IPA calculations from Table II with RRPA calculations of $\beta$ and $\gamma$ for the $3 s$ subshell of Ar and confirm the large value of $\gamma_{3 s}$ near threshold found in [5]. In the upper panel of the figure, both IPA and RRPA cross sections are shown. A deep "Cooper" minimum (where the $3 s \rightarrow p$ amplitude vanishes) is observed in the RRPA cross section near threshold, and is responsible for the fluxuations of $\gamma$ in this energy region. The corresponding Cooper minimum in IPA calculations is below threshold; correlation moves the minimum above threshold. A small departure of $\beta$ from the expected value of 2 is also found at the position of the Cooper minimum. (It may be difficult to observe the rapid variations of $\gamma$ in Ar owing to the smallness of the cross section in the energy region of interest.) The $\gamma$ parameters for the $4 s$ subshell of $\mathrm{Kr}$ and the $5 s$ subshell of Xe behave similarly near threshold; there are fluctuations associated with Cooper minima in the $n s \rightarrow p$ amplitudes. In these cases, however, the minima in both IPA and RRPA amplitudes occur above 

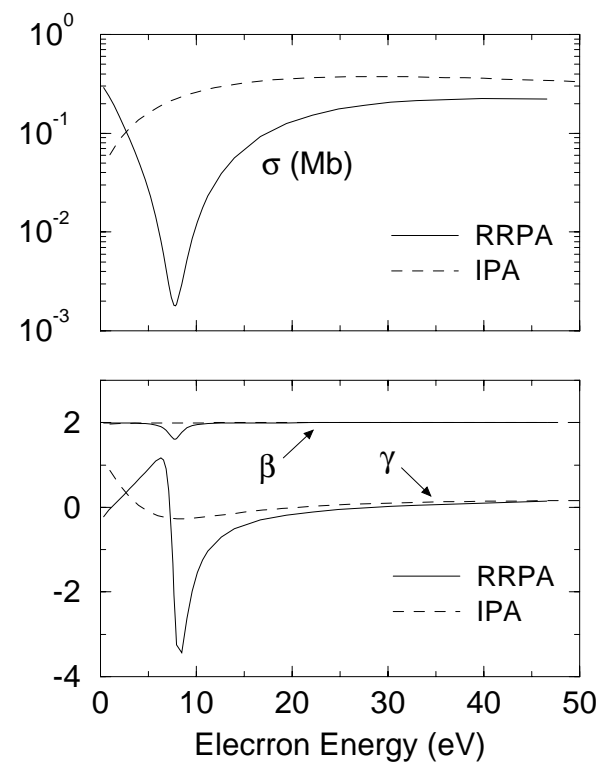

Figure 4: Left panel: Comparison of IPA and RRPA calculations for the Ar $3 s$ cross section $\sigma$. Right panel: Comparison of IPA and RRPA calculations of $\beta$ and $\gamma$ for the Ar $3 s$ shell.

threshold, so differences between IPA results shown in Table II and RRPA calculations are less dramatic. Results of detailed RRPA calculations for other low-energy cases will be published elsewhere.

\section{Acknowledgments}

The authors would like to thank D. Lindle, H. Gould and E. Kessler for useful discussions. The work of W. R. J. and A. D. was supported in part by NSF Grant No. Phy 99-70666. The work of K. T. C. was performed under the auspices of the U. S. Department of Energy by Lawrence Livermore National Laboratory under Contract No. W-7405-ENG-48. 


\section{References}

[1] M. Ya. Amusia and N. A. Cherepkov, Case Studies in Atomic Physics (North-Holland, Amsterdam, 1975), Vol. 5, p. 155.

[2] A. Bechler and R. H. Pratt, Phys. Rev. A 39, 1774 (1989); 42, 6400 (1990).

[3] J. H. Scofield, Phys. Rev. A 40, 7054 (1989); Physica Scripta 41, 59 (1990).

[4] J. W. Cooper, Phys. Rev. A 42, 6942 (1990); 47, 1841 (1993).

[5] M. Ya Amusia, A. S. Baltenkov, Z. Felfli, and A. Z. Msezane, Phys. Rev. A 59, R2544 (1999).

[6] B. Krässig, M. Jung, D. S. Gemmell, E. P. Kanter, T. LeBrun, S. H. Southworth, and L. Young, Phys. Rev. Lett. 75, 4736 (1995).

[7] M. Jung, B. Krässig, D. S. Gemmell, E. P. Kanter, T. LeBrun, S. H. Southworth, and L. Young, Phys. Rev. A 54, 2127 (1996).

[8] O. Hemmers, G. Fischer, P. Glans, D. L. Hansen, H. Wang, S. B. Whitfield, R. Wehlitz, J. C. Levin, I. A. Sellin, R. C. C. Perera, E. W. B. Dias, H. S. Chakraborty, P. C. Deshmukh, S. T. Manson, and D. W. Lindle, J. Phys. B 30, L727 (1997).

[9] W. R. Johnson, A. Derevianko, K. T. Cheng, V. K. Dolmatov, and S. T. Manson, Phys. Rev. A 59, 3609 (1999).

[10] A. Derevianko, O. Hemmers, S. Oblad, P. Glans, H. Wang, S. B. Whitfield, R. Wehlitz, I. A. Sellin, W. R. Johnson, and D. W. Lindle (in preparation) (1999).

[11] W. R. Johnson and C. D. Lin, Phys. Rev. A 20, 964 (1979); W. R. Johnson and K. T. Cheng, ibid. 20, 978 (1979).

[12] C. E. Moore, Atomic Energy Levels, Nat. Stand. Ref. Data Ser., Nat. Bur. Stand. (U.S.), 35 V. I-III (1971).

[13] R. D. Deslattes, J. E. Kessler, P. Indelicato and E. Lindroth, in International Tables for Crystallography, edited by T. Price (Kluwer Academic Publishers, London, 1997), Vol. C, pp. 11-22.

[14] P. Indelicato, B. Boucard and E. Lindroth, Eur. Phys. J, D 3, 29 (1998).

[15] K. D. Sevier, At. Data \& Nucl. Data Tables, 24, 323 (1979).

[16] K.-N. Huang, W. R. Johnson and K. T. Cheng, At. Data \& Nucl. Data Tables, 26, 33 (1981). 


\section{EXPLANATION OF TABLES}

TABLE I. Non-dipole Angular Distribution Parameters for Photoelectrons from the Subshells of $\mathrm{He}, \mathrm{Ne}, \mathrm{Ar}, \mathrm{Kr}$, and $\mathrm{Xe} \mathrm{(20-5000} \mathrm{eV).}$

Element symbol, ionized subshell, and binding energy given in heading.

$E_{b} \quad$ Electron binding energy $(\mathrm{eV})$ from Table A.

$E \quad$ Photoelectron energy $(\mathrm{eV})$.

$\omega \quad$ Photon energy, $\omega=E+E_{b}(\mathrm{eV})$.

$\beta \quad O(1)$ angular-distribution parameter in Eq. (1).

$\delta, \gamma \quad O(k a)$ angular-distribution parameters in Eq. (1).

$\Delta \beta, \lambda, \mu, \nu \quad O\left(k^{2} a^{2}\right)$ angular-distribution parameters in Eq. (1).

TABLE II. Non-dipole Angular Distribution Parameters for Photoelectrons from the Outer Subshells of Kr and Xe $(2-60 \mathrm{eV})$.

Element symbol, ionized subshell, and binding energy given in heading.

$\begin{array}{cl}E_{b} & \text { Electron binding energy }(\mathrm{eV}) \text { from Table A. } \\ E & \text { Photoelectron energy }(\mathrm{eV}) . \\ \omega & \text { Photon energy, } \omega=E+E_{b}(\mathrm{eV}) . \\ \beta & O(1) \text { angular-distribution parameter in Eq. (1). } \\ \delta, \gamma & O(k a) \text { angular-distribution parameters in Eq. (1). }\end{array}$

